electronic pro-forma for patients notes to assist with completing the relevant legal steps when implementing an EDC.

Conclusion. Our study identified a lack of confidence in understanding the MHA and completing an EDC. Our educational materials will provide an invaluable source of information for junior doctors, in particular those with little experience of the MHA. Importantly, our resources will ensure the legal aspects of implementing an EDC are both complied with and documented appropriately.

Prevalence, associated factors and prevention of burnout in psychiatry trainees in Central and North West London NHS Foundation Trust

Grace $\mathrm{Xia}^{*}$ and Ahrane Jayakumar

Central and North West London NHS Trust

${ }^{\star}$ Corresponding author.

doi: 10.1192/bjo.2021.206

Aims. To assess burnout, resilience, professional quality of life and coping mechanisms in Central and North West London psychiatry trainees

Objectives. To determine Key factors associated with stress and burnout in workplace Effects of burnout on patient care and doctors Coping mechanisms used by trainees

Background. Burnout is a well established condition that has been recently reported to affect a third of doctors. Psychiatrists in particular represents a high risk group among doctors for experiencing burnout, alcohol and drug use, posing suicide risk and other forms of work related stress.

Method. The study comprised of a cross sectional questionnaire survey which included measure of stress (General Health Questionnaire), burnout (Maslach Burnout Inventory), and satisfaction with medicine as a career and personality (Big Five). During October to December 2019, core trainee and specialty trainee doctors in CNWL were asked to complete an online survey via emails.

Result. We collected data from 50 CNWL psychiatry trainees. The sample consisted of 20 females (40\%) and 30 males (60\%). Ages varied from $26-58$ years old, with a median age of 28 . Core trainees (CT1-3) were recorded as $72 \%$ and specialty trainees at $28 \%$.

Of those who responded, around half of the trainees (52\%) experienced high levels of stress outside of work in their personal life. The most common causes that trainees felt makes psychiatry a stressful profession were violence and fear of violence, limited resources, dealing with confrontational patients, inability to affect systemic change and increasing culture of blame. Around half of respondents (54\%) felt that they have experienced burnout but only $26 \%$ of respondents knew where to go to find resources to help cope with burnout. Physical exercise and speaking to colleagues were the most common coping mechanisms used by trainees to deal with stress.

Free text responses on what can be improved in workplace to enhance a positive experience of work included improving multidisciplinary interactions, easily accessible resources and increasing staffing levels. $74 \%$ of respondents felt they continued to care about what happens to patients regardless of working conditions. Conclusion. Half of CNWL trainee doctors who responded have experienced burnout. Some factors associated with stress and burnout in doctors are unique to psychiatry profession. Free text responses were useful in identifying areas for improvement in work places and useful coping mechanisms, which can be used to inform prevention and implement interventions to tackle burnout.
Suicidality in patients with bipolar depression: findings from a lower middle-income country

Siqi Xue ${ }^{1 *}$, John Hodsoll ${ }^{2}$, Ameer Bukhsh Khoso ${ }^{3}$, Muhammad Omair Husain ${ }^{4}$, Imran B Chaudhry, Allan H Young ${ }^{2}$, Juveria Zaheer ${ }^{4}$, Nusrat Husain ${ }^{5}$ and Benoit Mulsant ${ }^{1}$

${ }^{1}$ University of Toronto; ${ }^{2}$ king's college london; ${ }^{3}$ Pakistan Institution of Living and Learning; ${ }^{4}$ Centre for Addiction and Mental Health and ${ }^{5}$ University of Manchester

${ }^{\star}$ Corresponding author.

doi: 10.1192/bjo.2021.207

Aims. Among low- and middle-income countries (LMICs), bipolar disorder is recognized as one of the leading causes of disease burden for adults and is associated with marked suicide risk. There are limited data on suicidal ideation in bipolar disorder from LMICs. This study presents cross-sectional data on the prevalence of suicidality and associated patient characteristics among patients with bipolar depression in Pakistan, a lower-middle income country and the fifth most populous country in the world.

Method. Participants were recruited through outpatient psychiatric clinics in between 2016-2019 in Karachi, Lahore, Hyderabad and Rawalpindi between 2016-2019. Participants were aged 18 to 65 years with a known diagnosis of bipolar disorder and currently in a depressive episode. Suicidality was assessed using the suicide item of the 17-item Hamilton Depression Rating Scale (HAM-D) and levels of severity were categorized as absent, mild/moderate, or severe. Biometric data and biomarkers were obtained. Descriptive statistics were used to describe prevalence and proportional odds regression models were applied to establish correlates to suicidal ideation.

Result. Among the 266 participants, $67 \%$ indicated suicidality of any level and $16 \%$ endorsed severe suicidality. Lower body mass index $(\mathrm{BMI})(\mathrm{OR}=0.93,95 \% \mathrm{CI}=0.88-0.98)$, higher HAM-D score $(\mathrm{OR}=1.29,95 \% \mathrm{CI}=1.16-1.43)$, lower $\mathrm{C}$-reactive protein (CRP) level ( $\mathrm{OR}=0.53,95 \% \mathrm{CI}=0.40-0.70)$, and increased number of inpatient hospitalizations $(\mathrm{OR}=1.16,95 \% \mathrm{CI}=1.03-1.31)$ were identified as significant predictors of suicidality in the fully adjusted regression model. No patient demographic data, including age, gender, marital status, socioeconomic status, and years of education were associated with severity of suicidality.

Conclusion. There exists a high prevalence of suicidal ideation among patients with bipolar depression in Pakistan. Our findings add to the limited literature on suicidality in bipolar disorder in the LMIC context and suggest roles of biological variables such as BMI and CRP level in predicting suicidal ideation and potentially suicidal behaviours in bipolar depression. More studies are needed to see whether such findings can be replicated in other similar LMIC settings, and to explore potential physiological pathways linking BMI, inflammatory biomarkers and suicidality in bipolar disorder.

\section{Implementation of treatment escalation plans in a community psychiatric hospital}

Anne Yan Ting Chua ${ }^{1 \star}$, Adnaan Ghanchi ${ }^{1}$, Jessica Grayston ${ }^{1}$, Nida Yasmeen ${ }^{2}$, Sean Insigne ${ }^{2}$, Stephen Woolford ${ }^{1}$, Sahan Wijayaweera ${ }^{2}$, Harnish P Patel ${ }^{2}$, Jay Amin ${ }^{1}$ and Sangeeta Makh ${ }^{1}$

${ }^{1}$ University of Southampton and ${ }^{2}$ Southern Health NHS Foundation Trust

${ }^{\star}$ Corresponding author.

doi: 10.1192/bjo.2021.208 
Aims. Treatment Escalation Plans (TEP) detail appropriate ceilings of care and guide treatment of patients based on shared decision making. Whilst established in many acute trusts, TEP are not frequently used in community mental health hospitals. This is particularly concerning in organic mental health wards, where patients with severe dementia may be transferred to acute hospitals for treatment without consideration about whether this is appropriate. Our aim for this quality improvement project was to develop and implement TEP within a community mental health hospital to support the management of our older patients with severe mental illness.

Method. We designed a TEP form based on a prototype used in a partner acute trust and evaluated its use on our wards, comprising 20 patients between August-September 2020. This included clear options of the different ceilings of care and what they comprised of for our patients. We obtained quantitative data on the use of TEP, including the length of time from admission to completion, as well as qualitative data from healthcare staff regarding their experience of using TEP.

Result. TEP implementation was feasible and well received among members of staff. All 20 patients had a TEP in place within 2 weeks of admission. The mean number of days taken to complete a TEP form in August-September was 7.1. A snapshot done 2 months later showed new admissions had a mean number of days to complete TEP reduced to 3.2. There was an improvement in understanding the purpose, comprehensiveness and location of TEP forms during their implementation. The key theme that arose from qualitative analysis of healthcare staff comments was that TEP forms provided clear guidance on the appropriateness of escalation of care.

Conclusion. TEP forms offer clear guidance to treating clinicians about the ceilings of care for patients. This also allows for open conversations with patients or their next of kins regarding ceiling of care. This is especially important in mental health inpatients with dementia, when escalation of treatment is not always appropriate. TEP were successfully implemented in our community mental health hospital and we plan further post-implementation evaluation. We intend to roll out the TEP form across our mental health trust and share findings globally to promote best practice.

Driving digitisation of the workforce. Junior doctor's digital devices rollout at South London and Maudsley NHS Foundation Trust: an early review of uptake, usage and effects

\section{Tharun Zacharia}

South London and Maudsley NHS Foundation Trust, IoPPN King's College London

doi: 10.1192/bjo.2021.209
Aims. A Quality Improvement Project (QIP) was completed at South London and Maudsley NHS Foundation Trust (SLaM) to provide access of Trust laptops and smartphones (digital devices) to training doctors.

The aim of the study was to assess the views of trainees before and after the rollout of digital devices. Also, to assess barriers of device uptake/usage.

Method. Trainee doctors were surveyed before mass release of digital devices. Trainees were surveyed again 2 months later, providing ample opportunity to request a device.

Correlated survey questions asked about training grade, expectation to request a digital device (and subsequent completed request), expected usage cases (and actual usage cases), expected benefits (and actual benefits), perceived importance of access to each device (before and after access) and barriers for device uptake. Also inquired were general comments about the project and actual lead time on device access.

Result. 110, mixed training grade, doctors participated in both surveys combined. There was a high demand for digital devices. Laptop requests were highest, with greater clarity of potential usage cases noted for a laptop. Laptops were perceived to be the more important device to a trainee.

Common usage cases for laptops were clinical work (in and out of working hours). There was also high usage around educational and audit/QIP activities. Smartphones were used only for clinical work (in and out of working hours).

Having access to each device was most beneficial in saving clinician time. Other sizable benefits included improvements in communication with patients and other professionals, as well as benefits toward patient safety. The laptop specifically also improved educational access.

A larger proportion of trainees requested a mobile phone than were previously expecting to. New ways of working due to the pandemic were noted to be motivating factors toward usage of devices.

The biggest barrier to device uptake was the delay from request to acquisition (often over 4 weeks).

Numerous respondents used personal devices for Trust related activities. This was more prevalent with smartphones, than with laptops.

Conclusion. Digital device rollout was a valued endeavour, with enhanced demand secondary to the pandemic. Primary usage and benefits supported clinical effectiveness. The primary access barrier was the delay from point of request to point of delivery. As SLaM, and the NHS as a whole, becomes increasingly digitised, this program is vital to allow the Trust to ensure the trainee workforce are digitally equipped to continue to deliver safe, effective and quality care. 